\title{
Explicit Constructions of Dense Common Hypercyclic Subspaces
}

\author{
By \\ Juan B. SeOANE-SEPÚLVEdA*
}

\begin{abstract}
We give an explicit construction of a dense infinite dimensional vector space of hypercyclic vectors for the weighted backward shift $T_{\lambda}(|\lambda|>1)$. We also develop a technique to construct common hypercyclic vectors for countable families of these operators. The techniques developed here do not rely on the Baire category theorem or any kind of existence proof, as do most approaches to this problem.
\end{abstract}

\section{$\S 1 . \quad$ Introduction and Preliminaries}

If $X$ denotes an infinite dimensional separable Banach space and $T: X \rightarrow$ $X$ is a bounded linear operator on $X$, we say that $x \in X$ is a hypercyclic vector for $T$ if its orbit, $\left\{T^{n} x: n \in \mathbb{N}\right\}$, is dense in $X$. If there exists such an $x \in X$ we call $T$ a hypercyclic operator.

The problem of characterizing certain families of hypercyclic operators has been intensively studied in the last twenty years. A very famous family of such operators is the weighted backward shift. Given $X$ an infinite dimensional Banach space, we will say that $X$ admits a weighted backward shift, $T_{\lambda}$, if $X$ has a Schauder basis, $\left(e_{i}\right)_{i}$, and the operator

$$
\begin{array}{ccc}
T_{\lambda}: & X & X \\
\sum_{n \geq 1} x_{n} e_{n} & \mapsto \sum_{n \geq 2} \lambda \cdot x_{n} e_{n-1}
\end{array}
$$

Communicated by H. Okamoto. Received April 22, 2005. Revised May 24, 2006. 2000 Mathematics Subject Classification(s): Primary 47A16; Secondary 47B37.

Key words: Hypercyclic subspaces, weighted shift, common hypercyclic vectors.

*Department of Mathematical Sciences. Kent State University. Kent, Ohio 44242, USA. e-mail: jseoane@math.kent.edu 
is well defined. In [11] Rolewicz proved that this operator is hypercyclic on $c_{0}$ or $l^{p}(1 \leq p<\infty)$ if and only if $|\lambda|>1$, i.e. $T_{\lambda}$ has a hypercyclic vector if and only if $|\lambda|>1$.

In this paper we study whether it is possible to explicitly construct such vectors. A recent approach to a similar problem was solved constructively in [2], where a hypercyclic vector for the chaotic operator

$$
\begin{aligned}
T_{\lambda, c}: C_{0}[0, \infty) & \longrightarrow C_{0}[0, \infty) \\
f(t) & \mapsto \lambda f(t+c)
\end{aligned}
$$

was constructed. Here $C_{0}[0, \infty)$ is the Banach space of continuous functions on $[0, \infty)$ that vanish at $\infty$, endowed with the standard norm $\|f\|=$ $\max _{t \in[0, \infty)}|f(t)|$.

Abakumov and Gordon ([1]) showed that $l^{2}$ contains a vector which is simultaneously hypercyclic for all weighted backward shifts of weight $|\lambda|>1$. Bès ([3]) showed that every hypercyclic operator on a (real or complex) locally convex space has a dense invariant hypercyclic vector manifold. In [6] it was shown that if $T$ is a continuous linear operator acting on a separable Banach space and $T$ verifies the hypercyclicity criterion, then the set of hypercyclic vectors for $T, H C(T)$, is spaceable (i.e., there is a closed infinite dimensional vector space $V$ with $V \backslash\{0\} \subset H C(T))$ if and only if the essential spectrum of $T$ intersects the closed unit disk. Recently, Grivaux ([5]) proved that if $\left(T_{n}\right)_{n}$ is a sequence of hypercyclic operators on an infinite dimensional separable Banach space $X$, then there exists a dense linear subspace which is hypercyclic for every operator $T_{n}$.

The hypercyclicity of weighted shift operators has been studied by several other authors (see, e.g., [4, 7, 9, 10, 12]), where the most common tool of approaching this problem is the Baire category theorem or a similar kind of existence proof. Here we give new and constructive proofs of some known results that don't rely on the Baire category theorem. We believe that the constructive approach given in this paper has not been used before.

From now on $X$ denotes any infinite dimensional Banach space with basis admitting a weighted backward shift, $\left(e_{i}\right)_{i}$ will denote its Schauder basis (with $\left\|e_{i}\right\|=1$ for every $\left.i\right), v[i]$ denotes the $i$-th coefficient of $v \in X$ with respect to the basis, and $T_{\lambda}$ will denote the weighted backward shift operator acting on $X$, with $|\lambda|>1$.

In Section 2 we start by giving an explicit construction of a dense hypercyclic subspace for $T_{\lambda}$ acting on $X$, proving, constructively, the following known result. 
Theorem 1.1. The set of hypercyclic vectors for the weighted backward shift $T_{\lambda}$ acting on $X$ is dense-lineable, i.e., there is a dense infinite dimensional vector space $V$, so that $V \backslash\{0\} \subset H C\left(T_{\lambda}\right)$.

The vector space constructed in the previous result cannot be closed in general since, in [8], Montes showed that the weighted backward shift operator does not have a closed hypercyclic subspace when acting on $c_{0}$ or $l^{p}, 1 \leq p<\infty$.

In Section 3 we also give a new and constructive proof of the following known result:

Theorem 1.2. Given $\left\{\left(T_{\lambda_{m}}\right)_{m \in \mathbb{N}}: \lambda_{m} \in(a, b)\right\}$ a countable family of weighted backward shifts acting on $X$, there is a common hypercyclic vector for all the elements in the family.

Finally, in Section 4, we include an analysis of the previous constructions as well as a remark about the complex case. Some new results on the complex case are also given.

\section{$\S 2 . \quad$ Dense Hypercyclic Subspaces}

In this section we construct a dense infinite dimensional vector subspace $V \subset X$ whose every non-zero element is a hypercyclic vector for $T_{\lambda}$. To do this we build a basis for $V$. Let us begin by taking $\left(z_{j}\right)_{j} \in c_{00}$ a dense sequence in $X$; let us also denote the set of prime numbers by $P=\left\{p_{1}, p_{2}, p_{3}, \ldots\right\}$. Now, for each $p_{i} \in P$, we define a new sequence $\left(y_{p_{i}^{j}}\right)_{j} \subset X$ verifying that, for every $j>0$,

$$
z_{j}=y_{p_{1}^{j}}=y_{p_{2}^{j}}=y_{p_{3}^{j}}=y_{p_{4}^{j}}=y_{p_{5}^{j}}=\ldots
$$

For example, for $p=3 \in P$, we would have that the sequence $\left(y_{3^{j}}\right)_{j}$ is given by

$$
y_{3}=z_{1}, y_{9}=z_{2}, y_{27}=z_{3}, y_{81}=z_{4}, \ldots
$$

It is clear that, for every $p=p_{i} \in P,\left\{y_{p^{j}}\right\}_{j>0}$ is also a dense sequence in $X$ with $\left\{y_{p^{j}}\right\}_{j>0} \in c_{00}$, since, for every $p \in P, y_{p^{j}}=z_{j}, j>0$. Given $p \in P$, we now construct $x_{p} \in X$ by defining it on intervals of the form $\left[0, k_{p}\right],\left[k_{p^{j}}, k_{p^{j}}+m_{p^{j}}\right]$, and $\left[k_{p^{j}}+m_{p^{j}}, k_{p^{j+1}}\right]$, for $j>0$, as follows:

$$
x_{p}[i]:=\left\{\begin{array}{cl}
g_{p}[i] & \text { if } \quad 0<i \leq k_{p}, \\
\left(\frac{y_{p^{j}}}{\lambda^{k_{p^{j}}}}\right)\left[i-k_{p^{j}}\right] & \text { if } \quad k_{p^{j}}<i \leq k_{p^{j}}+m_{p^{j}}, \\
0 & \text { if } \quad k_{p^{j}}+m_{p^{j}}<i \leq k_{p^{j+1}},
\end{array}\right.
$$


where the previous elements satisfy the following:

1. $\left(m_{p^{j}}\right)_{j}$ is any strictly increasing sequence of positive integers so that

$$
\left\|y_{p^{j}}-\tilde{y_{p^{j}}}\right\|<\frac{1}{2^{j}}
$$

for every $j>0$, where $\left(\tilde{y_{p^{j}}}\right)[i]=\left(y_{p^{j}}\right)[i]$ for $1 \leq i \leq m_{p^{j}}$ and $\left(\tilde{y_{p^{j}}}\right)[i]=0$ for $i>m_{p^{j}}$,

2. $\left(k_{n}\right)_{n \geq 2}$ is any strictly increasing sequence of positive integers with $k_{2}=2$ and also satisfying the following:

(a) For $j>1$,

$$
\frac{\sum_{i=1}^{\infty}\left|y_{p^{j}}[i]\right|}{|\lambda|^{k_{p^{j}}-k_{p^{j}-1}}}<\frac{1}{p \cdot j^{2}}
$$

and

(b) $k_{p^{j}}+m_{p^{j}}<k_{p^{j}+1}$ for each $j \geq 1$, and

3. $\left(g_{p}\right)_{p \in P} \in c_{00}$ is a dense sequence in $X$ with $g_{p}[i]=0$ for every $i>k_{p}$.

Firstly let us see that, for every $p \in P, x_{p} \in X$. For this it suffices to show that $\sum_{i=1}^{\infty}\left|x_{p}[i]\right|<\infty$. By (2.2) we obtain that

$$
\sum_{i=1}^{\infty}\left|x_{p}[i]\right| \leq \sum_{i=1}^{k_{p}}\left|g_{p}[i]\right|+\sum_{j=1}^{\infty}\left(\frac{\sum_{i=1}^{\infty}\left|y_{p^{j}}[i]\right|}{|\lambda|^{k_{p^{j}}}}\right)<\sum_{i=1}^{k_{p}}\left|g_{p}[i]\right|+\sum_{j=1}^{\infty} \frac{1}{p \cdot j^{2}}<\infty,
$$

and thus $x \in X$.

Secondly we need to show that, for every $p \in P, x_{p}$ is hypercyclic for $T_{\lambda}$. Since for any $n \in \mathbb{N},\left\{z_{\ell} \mid \ell \geq n\right\}$ is also dense in $X$, to see that the set of iterates is dense in $X$, it suffices to show that, for every $p \in P$,

$$
\left\|T_{\lambda}^{k_{p^{j}}} x_{p}-y_{p^{j}}\right\| \rightarrow 0 \quad \text { as } \quad j \rightarrow \infty .
$$

Using (2.1) and (2.2) we obtain

$$
\begin{aligned}
\left\|T_{\lambda}^{k_{p^{j}}} x_{p}-y_{p^{j}}\right\| & \leq \frac{1}{2^{j}}+|\lambda|^{k_{p^{j}}} \cdot \sum_{s=j+1}^{\infty}\left(\frac{\sum_{i=1}^{\infty}\left|y_{p^{s}}[i]\right|}{|\lambda|^{k_{p^{s}}}}\right) \\
& =\frac{1}{2^{j}}+\sum_{s=j+1}^{\infty}\left(\frac{\sum_{i=1}^{\infty}\left|y_{p^{s}}[i]\right|}{|\lambda|^{k_{p^{s}}-k_{p^{j}}}}\right) \\
& <\frac{1}{2^{j}}+\sum_{s=j+1}^{\infty} \frac{1}{p \cdot s^{2}} \rightarrow 0 \text { as } \quad j \rightarrow \infty .
\end{aligned}
$$


We claim that

$$
V=\operatorname{span}\left\{x_{p}: p \in P\right\}
$$

is an infinite dimensional vector space so that $V \backslash\{0\} \subset H C\left(T_{\lambda}\right)$. By construction it is clear that $V$ is infinite dimensional. Indeed, take any $m$ different vectors $x_{p_{1}}, x_{p_{2}}, \ldots, x_{p_{m}}$, where $p_{i} \in P$ for every $1 \leq i \leq m$ and, without loss, $p_{1}>p_{2}>\cdots>p_{m}$. Then, it is clear that the elements

$$
\left\{T^{k_{p_{1}}} x_{p_{1}}, T^{k_{p_{1}}} x_{p_{2}}, \ldots, T^{k_{p_{1}}} x_{p_{m}}\right\}
$$

are linearly independent (where $T=T_{1}$ ), since they do not share non-zero coordinates, and therefore the elements

$$
\left\{x_{p_{1}}, x_{p_{2}}, \ldots, x_{p_{m}}\right\}
$$

are also linearly independent. Thus the system $\left\{x_{p}: p \in P\right\}$ is free, and $V$ is infinite dimensional. Let us now see that $V \backslash\{0\} \subset H C\left(T_{\lambda}\right)$. Take any $v \in V \backslash\{0\}$, i.e.

$$
v=\alpha_{1} x_{p_{1}}+\alpha_{2} x_{p_{2}}+\cdots+\alpha_{m} x_{p_{m}},
$$

where $p_{i} \in P$ for every $1 \leq i \leq m$ and, without loss, $p_{1}>p_{2}>\cdots>p_{m}$. We can also suppose that no $\alpha_{j}$ is zero. We will see that $v / \alpha_{1}$ is a hypercyclic vector for $T_{\lambda}$, and we will be done. To show this we prove that

$$
\left\|T_{\lambda}^{k^{p_{1}^{j}}}\left(v / \alpha_{1}\right)-y_{p_{1}^{j}}\right\| \rightarrow 0 \quad \text { as } \quad j \rightarrow \infty .
$$

We have

$$
\begin{aligned}
\| T_{\lambda}^{k^{p_{1}^{j}}}\left(v / \alpha_{1}\right) & -y_{p_{1}^{j}}\|\leq\| T_{\lambda}^{k^{p_{1}^{j}}} x_{p_{1}}-y_{p_{1}^{j}} \| \\
& +\left\|T_{\lambda}^{{ }^{p_{1}^{j}}}\left(\frac{\alpha_{2}}{\alpha_{1}} x_{p_{2}}\right)\right\|+\cdots+\left\|T_{\lambda}^{{ }^{k}{ }^{j}}\left(\frac{\alpha_{m}}{\alpha_{1}} x_{p_{m}}\right)\right\| \\
& \leq\left\|T_{\lambda}^{{ }^{p_{1}^{j}}} x_{p_{1}}-y_{p_{1}^{j}}\right\|+A \cdot\left\|T_{\lambda}^{k^{p_{1}^{j}}} x_{p_{2}}\right\|+\cdots+A \cdot\left\|T_{\lambda}^{{ }^{p_{1}^{j}}} x_{p_{m}}\right\| \\
& =\left\|T_{\lambda}^{{ }^{k}{ }^{p_{1}^{j}}} x_{p_{1}}-y_{p_{1}^{j}}\right\|+A \cdot \sum_{i=2}^{m}\left\|T_{\lambda}^{{ }^{k}{ }^{p_{1}^{j}}} x_{p_{i}}\right\|,
\end{aligned}
$$

where $A=\max \left\{\left|\frac{\alpha_{j}}{\alpha_{1}}\right|: 2 \leq j \leq m\right\}$. As we saw above, $\left\|T_{\lambda}^{{ }^{p_{1}^{j}}} x_{p_{1}}-y_{p_{1}^{j}}\right\|$ goes to zero as $j \rightarrow \infty$, so let us examine the rest of the sum on the right hand side. For $2 \leq i \leq m$, call $N_{i, j}$ the first integer so that $p_{1}^{j}<p_{i}^{N_{i, j}}$. Now, by condition (2.2) we obtain: 


$$
\begin{aligned}
\sum_{i=2}^{m}\left\|T_{\lambda}^{k^{p_{1}^{j}}} x_{p_{i}}\right\| & \leq \sum_{i=2}^{m}\left(|\lambda|^{k_{p_{1}^{j}}} \cdot \sum_{n=N_{i, j}}^{\infty} \frac{\sum_{q=1}^{\infty}\left|y_{p_{i}^{n}}[q]\right|}{|\lambda|^{k_{p_{i}^{n}}}}\right) \\
& =\sum_{i=2}^{m}\left(\sum_{n=N_{i, j}}^{\infty} \frac{\sum_{q=1}^{\infty}\left|y_{p_{i}^{n}}[q]\right|}{|\lambda|^{k_{p_{i}^{n}}-k_{p_{1}^{j}}}}\right) \\
& <\sum_{i=2}^{m}\left(\sum_{n=N_{i, j}}^{\infty} \frac{1}{p_{i} \cdot n^{2}}\right)<\sum_{i=2}^{m}\left(\sum_{n=N_{i, j}}^{\infty} \frac{1}{n^{2}}\right) \longrightarrow 0
\end{aligned}
$$

as $j \rightarrow \infty$ since, for every $2 \leq i \leq m, N_{i, j} \rightarrow \infty$ as $j \rightarrow \infty$. Therefore $v / \alpha_{1}$ is a hypercyclic vector for $T_{\lambda}$, and so is $v$. Thus we have constructed an infinite dimensional vector space whose every non-zero element is hypercyclic for $T_{\lambda}$. Let us now see that $V$ is dense in $X$. First notice that, for every $p \in P$,

$$
\left\|x_{p}-g_{p}\right\| \leq \sum_{j=1}^{\infty}\left(\frac{\sum_{i=1}^{\infty}\left|y_{p^{j}}[i]\right|}{|\lambda|^{k_{p^{j}}}}\right)<\sum_{j=1}^{\infty} \frac{1}{p \cdot j^{2}}=\frac{\pi^{2}}{6 p} .
$$

Next, take any $z \in X$ and any $\varepsilon>0$. Now, take $p$ prime, big enough, so that $\frac{\pi^{2}}{6 p}<\frac{\varepsilon}{2}$. Let $q$ be a prime number with $q>p$ and with $\left\|g_{q}-z\right\|<\frac{\varepsilon}{2}$. Then, we obtain

$$
\left\|x_{q}-z\right\| \leq\left\|x_{q}-g_{q}\right\|+\left\|g_{q}-z\right\|<\frac{\pi^{2}}{6 q}+\frac{\varepsilon}{2}<\frac{\pi^{2}}{6 p}+\frac{\varepsilon}{2}<\varepsilon,
$$

thus $\left\{x_{p}\right\}_{p \in P}$ is dense in $X$. Therefore $V$ is a dense infinite dimensional vector subspace $V \subset X$ whose every non-zero element is a hypercyclic vector for $T_{\lambda}$.

\section{§3. Common Hypercyclic Vectors for Families of Operators}

In this section we will describe the construction of common hypercyclic vectors for countable families of weighted backward shift operators. For that, let us take $1<a<b<\infty$, and consider the rational numbers in $(a, b),\left(\lambda_{m}\right)_{m}$. In order to construct a vector $x \in X$, hypercyclic for each $T_{\lambda_{m}}$, let us denote $\left(y_{j}\right)_{j} \in c_{00}$ a dense sequence in $X$. We also need to define the following double sequence, $\{p(j, m)\}_{j, m \in \mathbb{N}}$, given by

$$
p(j, m)=\frac{1}{2} \cdot(j+m-2) \cdot(j+m-1)+m-1 \quad \text { for } \quad j, m \geq 1 .
$$

To see that $\{p(j, m)\}_{j, m \in \mathbb{N}}$ covers all $\mathbb{N} \cup\{0\}$ in a one to one fashion, it is enough to picture the infinite matrix of elements $p(j, m)$, as also appears in [6], i.e. 


$$
\left(\begin{array}{ccccc}
0 & 2 & 5 & 9 & \cdots \\
1 & 4 & 8 & 13 & \cdots \\
3 & 7 & 12 & 18 & \cdots \\
6 & 11 & 17 & 24 & \cdots \\
\vdots & \vdots & \vdots & \vdots & \ddots
\end{array}\right)
$$

As we did before, we now define some elements that we need in our construction. The following elements look similar to those we used before, but it is necessary to repeat them here in order to avoid confusion:

1. $\left(M_{n}\right)_{n}$ stands for any strictly increasing sequence of positive integers satisfying

$$
\left\|y_{j}-\tilde{y_{j, \ell}}\right\|<\frac{1}{2^{j+\ell}}
$$

for every $j, \ell \geq 1$, where $\left(\tilde{y_{j}, \ell}\right)[i]=\left(y_{j}\right)[i]$ for $1 \leq i \leq M_{p(j, \ell)}$ and $\left(\tilde{y_{j}, \ell}\right)[i]=$ 0 for $i>M_{p(j, \ell)}$; i.e., $\tilde{y_{j, \ell}}=\left(y_{j}^{1}, y_{j}^{2}, y_{j}^{3}, \ldots, y_{j}^{M_{p(j, \ell)}}, 0,0,0, \ldots\right)$.

2. We also take $\left(k_{j}\right)_{j}$ a strictly increasing sequence of positive integers with $k_{0}=0$, and satisfying the following conditions:

(a) For $j>0$,

$$
\left(\sum_{i=1}^{\infty}\left|y_{s}[i]\right|\right) \cdot \frac{b^{k_{j-1}}}{a^{k_{j}}}<\frac{1}{2^{s+t}}, \quad \text { where } \quad p(s, t)=j
$$

and

(b) $k_{j}+M_{j}<k_{j+1}$ for each $j \geq 0$.

We construct $x \in X$ by defining it on intervals of the form $\left[k_{p(j, m)}, k_{p(j, m)}\right.$ $\left.+M_{p(j, m)}\right]$ and $\left[k_{p(j, m)}+M_{p(j, m)}, k_{p(j, m)+1}\right]$ for every $j, m \geq 1$ as follows:

$$
x[i]:= \begin{cases}\left(\frac{1}{\lambda_{m}^{k_{p(j, m)}}} \cdot y_{j}\right)\left[i-k_{p(j, m)}\right] & \text { if } \quad k_{p(j, m)}<i \leq k_{p(j, m)}+M_{p(j, m)}, \\ 0 & \text { if } \quad k_{p(j, m)}+M_{p(j, m)}<i \leq k_{p(j, m)+1} .\end{cases}
$$

It may be helpful to picture $x$ as in the following diagram:

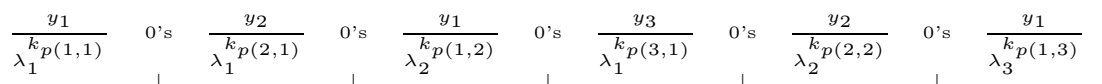

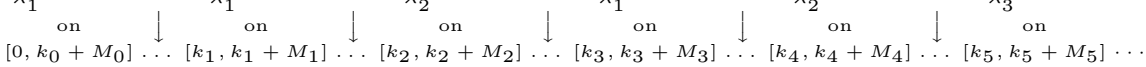


By condition (3.2),

$$
\begin{aligned}
\sum_{i=1}^{\infty}|x[i]| & \leq \sum_{t=1}^{\infty}\left(\sum_{s=1}^{\infty} \frac{\sum_{i=1}^{\infty}\left|y_{s}[i]\right|}{\lambda_{t}^{k_{p(s, t)}}}\right) \\
& <\sum_{t=1}^{\infty}\left(\sum_{s=1}^{\infty} \frac{1}{2^{s+t}}\right)=\left(\sum_{s=1}^{\infty} \frac{1}{2^{s}}\right)\left(\sum_{t=1}^{\infty} \frac{1}{2^{t}}\right)=1
\end{aligned}
$$

so $x \in X$. Next, to see that $x$ is hypercyclic for each $T_{\lambda_{m}}$, we show that, for each $m \in \mathbb{N}$,

$$
\left\|T_{\lambda_{m}}^{k_{p(j, m)}} x-y_{j}\right\| \longrightarrow 0 \quad \text { as } \quad j \rightarrow \infty .
$$

Using (3.1) we obtain that, for every $m \in \mathbb{N}$,

$$
\begin{aligned}
& \left\|T_{\lambda_{m}}^{k_{p(j, m)}} x-y_{j}\right\|<\frac{1}{2^{j+m}} \\
& +\lambda_{m}^{k_{p(j, m)}} \cdot \sum_{s=1}^{j-1} \frac{\sum_{i=1}^{\infty}\left|y_{s}[i]\right|}{\lambda_{j+m-s}^{k_{p(s, j+m-s)}}}+\lambda_{m}^{k_{p(j, m)}} \cdot \sum_{s=1}^{j+m-1}\left(\sum_{i=1}^{\infty}\left|y_{s}[i]\right| \cdot \sum_{t=j+m+1-s}^{\infty} \frac{1}{\lambda_{t}^{k_{p(s, t)}}}\right) \\
& +\lambda_{m}^{k_{p(j, m)}} \cdot \sum_{s=j+m}^{\infty}\left(\sum_{i=1}^{\infty}\left|y_{s}[i]\right| \cdot \sum_{t=1}^{\infty} \frac{1}{\lambda_{t}^{k_{p(s, t)}}}\right):=\frac{1}{2^{j+m}}+\sigma_{1}+\sigma_{2}+\sigma_{3} .
\end{aligned}
$$

We have that, for every $m \geq 1, \frac{1}{2^{j+m}} \rightarrow 0$ as $j \rightarrow \infty$. We now examine the values of $\sigma_{1}, \sigma_{2}$ and $\sigma_{3}$ as $j \rightarrow \infty$. Again, by (3.2),

$$
\begin{aligned}
\text { - } \sigma_{1} & <\sum_{s=1}^{j-1}\left[\left(\sum_{i=1}^{\infty}\left|y_{s}[i]\right|\right) \cdot \frac{b^{k_{p(j, m)}}}{a^{k_{p(s, j+m-s)}}}\right]<\sum_{s=1}^{j-1} \frac{1}{2^{j+m}}=\frac{j-1}{2^{j+m}}, \\
\text { - } \sigma_{2} & <\sum_{s=1}^{j+m-1}\left[\left(\sum_{i=1}^{\infty}\left|y_{s}[i]\right|\right) \cdot \sum_{t=j+m+1-s}^{\infty} \frac{b^{k_{p(j, m)}}}{a^{k_{p(s, t)}}}\right] \\
& <\sum_{s=1}^{j+m-1}\left(\sum_{t=j+m+1-s}^{\infty} \frac{1}{2^{s+t}}\right)=\frac{j+m-1}{2^{j+m}}, \\
\text { - } \sigma_{3} & <\sum_{s=j+m}^{\infty}\left(\sum_{i=1}^{\infty}\left|y_{s}[i]\right| \cdot \sum_{t=1}^{\infty} \frac{b^{k_{p(j, m)}}}{a^{k_{p(s, t)}}}\right)<\sum_{s=j+m}^{\infty}\left(\sum_{t=1}^{\infty} \frac{1}{2^{s+t}}\right)=\frac{1}{2^{j+m-1}}
\end{aligned}
$$

Thus $\sigma_{i} \rightarrow 0 \quad$ as $\quad j \rightarrow \infty$, for $i=1,2,3$. So, for each $m \geq 1$,

$$
\left\|T_{\lambda_{m}}^{k_{p(j, m)}} x-y_{j}\right\| \longrightarrow 0 \quad \text { as } \quad j \rightarrow \infty,
$$

and hence $x$ is a common hypercyclic vector for the family $\left\{T_{\lambda_{m}}\right\}_{m \in \mathbb{N}}$. 


\section{§4. Conclusions and Remarks on the Complex Case}

In this section we give an analysis of the previous constructions as well as some remarks about the complex case. Some new results on the complex case will be given as well (Propositions 4.1 and 4.2). So far, and to summarize, we have developed a technique that allowed us to give new and constructive proofs of the following known facts:

Theorem 4.1. Let $X$ be an infinite dimensional Banach space admitting a weighted backward shift $T_{\lambda}$, where $|\lambda|>1$. Then the set of hypercyclic vectors for the weighted backward shift is dense-lineable, i.e., there is a dense infinite dimensional vector space $V$, so that $V \backslash\{0\} \subset H C\left(T_{\lambda}\right)$.

and

Theorem 4.2. $\quad$ Let $X$ be an infinite dimensional Banach space admitting a weighted backward shift. Then, given $\left\{\left(T_{\lambda_{m}}\right)_{m \in \mathbb{N}}: \lambda_{m} \in(a, b)\right\}$ a countable family of weighted backward shifts acting on $X$, there is a common hypercyclic vector for all the elements in the family.

This last result was proved by showing that $\left(\left\|T_{\lambda_{m}}^{k_{p(j, m)}} x-y_{j}\right\|\right)_{j} \in c_{0}$ for every $m \in \mathbb{N}$. But we could wonder whether this vector $x$ could be also hypercyclic for some weighted backward shift $T_{r}$, where $r \in(a, b) \backslash\left\{\lambda_{m}: m \in\right.$ $\mathbb{N}\}$. We know that, for every $m \geq 1$,

$$
\lim _{j \rightarrow \infty}\left\|T_{\lambda_{m}}^{k_{p(j, m)}} x-y_{j}\right\|=0 .
$$

Now we show that, if $r \in(a, b) \backslash\left\{\lambda_{n}: n \in \mathbb{N}\right\}$, then the sequence $\left(\left\|T_{r}^{k_{p(j, m)}} x-y_{j}\right\|\right)_{j}$ is not convergent. In fact, we will see that there exists a subsequence $\left(N_{j}\right)_{j} \subset \mathbb{N}$ so that

$$
\lim _{j \rightarrow \infty}\left\|T_{r}^{k_{p\left(N_{j}, m\right)}} x-y_{N_{j}}\right\|=\infty .
$$

In order to show this, we first need to find a lower bound for $\left\|T^{k_{p(j, m)}} x\right\|$, where $T=T_{1}$. By construction, using the reverse triangle inequality twice and by (3.1), we have that

$$
\left\|T^{k_{p(j, m)}} x\right\| \geq \frac{\left\|\tilde{y_{j, m}}\right\|}{\lambda_{m}^{k_{p(j, m)}}}-\delta_{j} \geq \frac{\left\|y_{j}\right\|-\| y_{j}-\tilde{y_{j, m} \|}}{\lambda_{m}^{k_{p(j, m)}}}-\delta_{j} \geq \frac{\left\|y_{j}\right\|-\frac{1}{2^{j+m}}}{\lambda_{m}^{k_{p(j, m)}}}-\delta_{j},
$$

where $\delta_{j}=\left\|\sum_{i=k_{p(j, m)}+1}^{\infty} x\right\|$, the norm of the tail of $x$, and satisfies $\delta_{j} \rightarrow 0$ as $j \rightarrow \infty$. Consequently,

$$
\left\|T_{\lambda_{m}}^{k_{p(j, m)}} x\right\|=\lambda_{m}^{k_{p(j, m)}} \cdot\left\|T^{k_{p(j, m)}} x\right\| \geq\left\|y_{j}\right\|-\frac{1}{2^{j+m}}-\lambda_{m}^{k_{p(j, m)}} \cdot \delta_{j},
$$


where

$$
\lambda_{m}^{k_{p(j, m)}} \cdot \delta_{j} \leq \sigma_{1}+\sigma_{2}+\sigma_{3} \rightarrow 0 \text { as } j \rightarrow \infty,
$$

as we saw in the previous section. Take now any $r \in(a, b) \backslash\left\{\lambda_{n}: n \in \mathbb{N}\right\}$ and any $\lambda_{m}<r$. We want to investigate the behavior of $\left(\left\|T_{r}^{k_{p(j, m)}} x-y_{j}\right\|\right)_{j}$. The reverse triangle inequality gives that

$$
\left\|T_{r}^{k_{p(j, m)}} x-y_{j}\right\| \geq\left\|T_{r}^{k_{p(j, m)}} x-T_{\lambda_{m}}^{k_{p(j, m)}} x\right\|-\left\|T_{\lambda_{m}}^{k_{p(j, m)}} x-y_{j}\right\|,
$$

where $\left\|T_{\lambda_{m}}^{k_{p(j, m)}} x-y_{j}\right\| \rightarrow 0$ as $j \rightarrow \infty$. On the other hand, by using (4.1) and some calculations,

$$
\begin{aligned}
& \left\|T_{r}^{k_{p(j, m)}} x-T_{\lambda_{m}}^{k_{p(j, m)}} x\right\|=\left|\lambda_{m}^{k_{p(j, m)}}-r^{k_{p(j, m)}}\right| \cdot\left\|T^{k_{p(j, m)}} x\right\| \\
& =\left|\lambda_{m}-r\right| \cdot\left(\sum_{q=1}^{k_{p(j, m)}} r^{q-1} \cdot \lambda_{m}^{k_{p(j, m)}-q}\right) \cdot\left\|T^{k_{p(j, m)}} x\right\| \\
& \geq\left|\lambda_{m}-r\right| \cdot \lambda_{m}^{k_{p(j, m)}-1} \cdot k_{p(j, m)} \cdot\left(\frac{\left\|y_{j}\right\|-\frac{1}{2^{j+m}}}{\lambda_{m}^{k_{p(j, m)}}}-\delta_{j}\right) \\
& =\left(\frac{\left|\lambda_{m}-r\right|}{\lambda_{m}}\right) \cdot k_{p(j, m)} \cdot\left(\left\|y_{j}\right\|-\frac{1}{2^{j+m}}-\lambda_{m}^{k_{p(j, m)}} \cdot \delta_{j}\right) .
\end{aligned}
$$

Now, using the previous inequality and (4.4), we obtain

$$
\begin{aligned}
\left\|T_{r}^{k_{p(j, m)}} x-y_{j}\right\| & \geq \frac{\left|\lambda_{m}-r\right|}{\lambda_{m}} \cdot k_{p(j, m)} \cdot\left(\left\|y_{j}\right\|-\frac{1}{2^{j+m}}-\lambda_{m}^{k_{p(j, m)}} \cdot \delta_{j}\right) \\
& -\left\|T_{\lambda_{m}}^{k_{p(j, m)}} x-y_{j}\right\| .
\end{aligned}
$$

Next, since $\left(y_{j}\right)_{j}$ is dense in $X$, choose a subsequence $\left(N_{j}\right)_{j} \subset \mathbb{N}$ so that $\inf _{j}\left\|y_{N_{j}}\right\| \geq 1$. Thus, since $k_{p\left(N_{j}, m\right)} \rightarrow \infty$ as $j \rightarrow \infty$ and, by the previous inequality together with (4.3), we obtain

$$
\left\|T_{r}^{k_{p\left(N_{j}, m\right)}} x-y_{N_{j}}\right\| \rightarrow \infty \text { as } j \rightarrow \infty .
$$

Hence the sequence $\left(\left\|T_{r}^{k_{p(j, m)}} x-y_{j}\right\|\right)_{j}$ is not convergent. We summarize these last results in the following proposition.

Proposition 4.1. The sequence $\left(\left\|T_{r}^{k_{p(j, m)}} x-y_{j}\right\|\right)_{j}$ is convergent if and only if $r \in\left\{\lambda_{m}: m \in \mathbb{N}\right\}$ and, in this case, its limit is 0 . Moreover, if $r \in(a, b) \backslash\left\{\lambda_{m}: m \in \mathbb{N}\right\}$ then the sequence $\left(\left\|T_{r}^{k_{p(j, m)}} x-y_{j}\right\|\right)_{j}$ is unbounded. 
However, we do not know whether the vector constructed in the previous section is also hypercyclic for every member of $\left\{T_{r}: r \in(a, b)\right\}$.

We could also wonder what happens in the complex case, i.e. what is the behavior of the sequence $\left(\left\|T_{r}^{k_{p(j, m)}} x-y_{j}\right\|\right)_{j}$ when $r$ is of the form $\lambda_{m} \cdot e^{i \theta}$ for some $\theta \in \mathbb{R}$. In this case we claim that this sequence has a zero subsequence. To see this let us choose $\left(y_{N_{j}}\right)_{j}$, a subsequence of $\left(y_{j}\right)_{j}$, converging to zero. Then, using the same notation as before and equation (3.1), we obtain

$$
\begin{aligned}
\left\|T_{\lambda_{m} \cdot e^{i \theta}}^{k_{p\left(N_{j}, m\right)} x} x-y_{N_{j}}\right\| \leq & \left\|\frac{\left(\lambda_{m} \cdot e^{i \theta}\right)^{k_{p\left(N_{j}, m\right)}}}{\lambda_{m}^{k_{p\left(N_{j}, m\right)}}} \cdot \tilde{y_{N_{j}, m}}-y_{N_{j}}\right\|+\lambda_{m}^{k_{p\left(N_{j}, m\right)}} \cdot \delta_{N_{j}} \\
\leq & \left\|e^{i \theta k_{p\left(N_{j}, m\right)}} \cdot y_{N_{j}, m}-\tilde{y_{N_{j}, m}}\right\|+\left\|y_{N_{j}}-y_{N_{j}, m}\right\| \\
& +\lambda_{m}^{k_{p\left(N_{j}, m\right)}} \cdot \delta_{N_{j}} \\
\leq & \left|e^{i \theta k_{p\left(N_{j}, m\right)}}-1\right| \cdot\left\|y_{N_{j}}\right\|+\frac{1}{2^{N_{j}+m}}+\lambda_{m}^{k_{p\left(N_{j}, m\right)}} \cdot \delta_{N_{j}} \\
\leq & 2 \cdot\left\|y_{N_{j}}\right\|+\frac{1}{2^{N_{j}+m}}+\lambda_{m}^{k_{p\left(N_{j}, m\right)}} \cdot \delta_{N_{j}} \longrightarrow 0 \text { as } \quad j \rightarrow \infty .
\end{aligned}
$$

In particular, if $\frac{\theta}{2 \pi} \in \mathbb{Z}$, we get the original situation, and the sequence itself converges to zero. On the other hand, the sequence $\left\|T_{\lambda_{m} \cdot e^{i \theta}}^{k_{p\left(N_{j}, m\right)}} x-y_{N_{j}}\right\|$ has a divergent subsequence if $\frac{\theta}{2 \pi} \notin \mathbb{Z}$. Indeed, take $\left(y_{M_{j}}\right)_{j}$ a subsequence of $\left(y_{j}\right)_{j}$ with $\left\|y_{M_{j}}\right\| \rightarrow \infty$ as $j \rightarrow \infty$, and any $\theta \in \mathbb{R}$ with $\frac{\theta}{2 \pi} \notin \mathbb{Z}$. Proceeding as above and using the reverse triangle inequality together with (3.1) we obtain

$$
\begin{aligned}
\left\|T_{\lambda_{m} \cdot e^{i \theta}}^{k_{p\left(M_{j}, m\right)}} x-y_{M_{j}}\right\| \geq & \left\|\frac{\left(\lambda_{m} \cdot e^{i \theta}\right)^{k_{p\left(M_{j}, m\right)}}}{\lambda_{m}^{k_{p\left(M_{j}, m\right)}}} \cdot y_{M_{j}, m}-y_{M_{j}, m}\right\| \\
& -\left(\left\|y_{M_{j}}-y_{M_{j}, m}\right\|+\lambda_{m}^{k_{p\left(M_{j}, m\right)}} \cdot \delta_{M_{j}}\right) \\
> & \mid e^{i \theta k_{p\left(M_{j}, m\right)}-1 \mid \cdot\left\|y_{M_{j}, m}\right\|} \\
& -\left(\frac{1}{2^{M_{j}+m}}+\lambda_{m}^{k_{p\left(M_{j}, m\right)}} \cdot \delta_{M_{j}}\right) .
\end{aligned}
$$

And, since $\frac{1}{2^{M_{j}+m}}+\lambda_{m}^{k_{p\left(M_{j}, m\right)}} \cdot \delta_{M_{j}} \rightarrow 0$ as $j \rightarrow \infty$, we have that $\| T_{\lambda_{m} \cdot e^{i \theta}}^{k_{p\left(M_{j}, m\right)}} x-$ $y_{M_{j}} \| \rightarrow \infty$ as $j \rightarrow \infty$. So we can conclude that

Proposition 4.2. Given the sequence $\left(\left\|T_{\lambda_{m} \cdot e^{i \theta}}^{k_{p(j)}} x-y_{j}\right\|\right)_{j}$, we have the following:

1. It is convergent if and only if $\frac{\theta}{2 \pi} \in \mathbb{Z}$ and, in such case, its limit is 0 .

2. For every $\theta \in \mathbb{R}$ it contains a subsequence converging to 0 . 
To conclude this paper we mention that the techniques of Sections 2 and 3 can be combined in order to construct dense common hypercyclic subspaces for sequences of weighted backward shifts.

\section{Acknowledgement}

The author would like to thank Richard M. Aron for fruitful conversations and comments on the manuscript.

\section{References}

[1] E. Abakumov and J. Gordon, Common hypercyclic vectors for multiples of backward shift, J. Funct. Anal. 200 (2003), no. 2, 494-504.

[2] R. M. Aron, J. B. Seoane-Sepúlveda and A. Weber, Chaos on function spaces, Bull. Austral. Math. Soc. 71 (2005), no. 3, 411-415.

[3] J. P. Bès, Invariant manifolds of hypercyclic vectors for the real scalar case, Proc. Amer. Math. Soc. 127 (1999), no. 6, 1801-1804.

[4] G. Godefroy and J. H. Shapiro, Operators with dense, invariant, cyclic vector manifolds, J. Funct. Anal. 98 (1991), no. 2, 229-269.

[5] S. Grivaux, Construction of operators with prescribed behaviour, Arch. Math. (Basel) 81 (2003), no. 3, 291-299.

[6] M. González, F. León-Saavedra and A. Montes-Rodríguez, Semi-Fredholm theory: hypercyclic and supercyclic subspaces, Proc. London Math. Soc. (3) 81 (2000), no. 1, 169-189.

[7] F. León-Saavedra and A. Montes-Rodríguez, Spectral theory and hypercyclic subspaces, Trans. Amer. Math. Soc. 353 (2001), no. 1, 247-267 (electronic).

[8] A. Montes-Rodríguez, Banach spaces of hypercyclic vectors, Michigan Math. J. 43 (1996), no. 3, 419-436.

[9] A. Montes-Rodríguez and H. N. Salas, Supercyclic subspaces: spectral theory and weighted shifts, Adv. Math. 163 (2001), no. 1, 74-134.

[10] , Supercyclic subspaces, Bull. London Math. Soc. 35 (2003), no. 6, 721-737.

[11] S. Rolewicz, On orbits of elements, Studia Math. 32 (1969), 17-22.

[12] H. N. Salas, A hypercyclic operator whose adjoint is also hypercyclic, Proc. Amer. Math. Soc. 112 (1991), no. 3, 765-770. 